\title{
Distribución, abundancia relativa y alimentación de pintarroja Schroederichthys bivius Müller \& Henle, 1838 en el Océano Atlántico sudoccidental
}

\author{
Distribution, relative abundance and feeding of narrow-mouthed catshark Schroederichthys \\ bivius Müller \& Henle, 1838 in the Southwest Atlantic Ocean \\ Felisa Sánchez $^{1}$, Noemí R. Marí1 ${ }^{1}$ Juan C. Bernardele ${ }^{1}$ \\ ${ }^{1}$ Instituto Nacional de Investigación Pesquera (INIDEP), Paseo Victoria Ocampo N¹, (B7602HSA), \\ Mar del Plata, Buenos Aires, Argentina \\ feliza@inidep.edu.ar
}

\begin{abstract}
The narrow-mouthed catshark, Schroederichthys bivius, is a cartilaginous fish that inhabits the Southwest Atlantic Ocean ranging from $34^{\circ} \mathrm{S}$ to $54^{\circ} \mathrm{S}$ at depths from 45 to $350 \mathrm{~m}$. Like other chondrichthyans, it is caught as by-catch in fisheries of other target species, in research cruises as well as in commercial fleet. Information from 1924 fishing trawls of research cruises performed for INIDEP, carried out from 1996 to 2006, was analysed. Data was obtained in two areas: northward (PBN) and southward (PPA), both in different seasons. Biomass values estimated in PPA were three times superior to PBN area. S. bivius presented a wide distribution without seasonal variations but its frequency of apearance and its relative abundance showed a southward increasing trend. Values of relative abundance were between 0.010 and $9.1 \mathrm{t} \mathrm{mn}^{-2}$, being $97 \%$ of these values lower than $3.0 \mathrm{t} \mathrm{mn}^{-2}$. In order to determine feeding habits a total of 736 stomachs were analysed. A total of $88 \%$ of sampled individuals evidenced food ingestion. General trophic spectrum consisted on cephalopods (39\%), fishes (36\%), benthic crustaceans (18\%), other benthic invertebrates (6\%) and gelatinous zooplankton (1\%). In PBN, fishes were principal prey whereas cephalopods were in PPA. Although no differences were observed in diet composition between males and females, a diet shift was observed near 64 cm TL. General trophic level estimated was 3.25.
\end{abstract}

Key words: Argentine sea, chondrichthyans, trophic spectrum, trophic level

\section{Introducción}

Los peces cartilaginosos poseen ciertas características biológicas que los hacen altamente vulnerables a las pesquerías de gran escala (Pratt \& Casey 1990). Asimismo, al ser considerados 'captura incidental', se dificulta el manejo de sus pesquerías, por lo que generalmente, los signos de declinación en las capturas de cada especie no son advertidos oportunamente (Van Der Molen et al. 1998,
Resumen.- La pintarroja, Schroederichthys bivius, es un pez cartilaginoso que se distribuye en el Atlántico sudoccidental, entre las latitudes $34^{\circ} \mathrm{S}$ y $54^{\circ} \mathrm{S}$, y a profundidades entre $45 \mathrm{y}$ $350 \mathrm{~m}$. Como otros condrictios, constituye parte de la denominada 'captura incidental' en las capturas de especies objetivo, tanto en campañas de investigación como en la flota comercial. Se analizó información de 1924 lances de pesca de campañas de investigación del INIDEP, obtenidas entre 1996 y 2006. Los datos provinieron de dos áreas: norte (PBN) y sur (PPA) y de diferentes épocas del año. Las biomasas registradas en PPA fueron hasta tres veces superiores que en PBN. S. bivius presentó una amplia distribución que no varió estacionalmente, pero su frecuencia de aparición y su abundancia relativa aumentaron hacia el sur. Los valores de abundancia relativa oscilaron entre 0,010 y $9,1 \mathrm{t} \mathrm{mn}^{-2}$, y el $97 \%$ de los valores fue inferior a 3,0 $\mathrm{t} \mathrm{mn}^{-2}$. Para el análisis trófico se revisaron 736 estómagos. El 88\% de los individuos muestreados presentó contenido en sus estómagos. Su espectro trófico general estuvo integrado por cefalópodos (39\%), peces (36\%), crustáceos bentónicos (18\%), otros invertebrados bentónicos (6\%) y zooplancton gelatinoso (1\%). En PBN, la presa principal fueron los peces y en PPA los cefalópodos. Si bien, no se observaron diferencias en la alimentación entre machos y hembras, se encontró un cambio en la dieta de la pintarroja alrededor de los $64 \mathrm{~cm}$ de longitud total. El nivel trófico general estimado fue de 3,25.

Palabras clave: Mar Argentino, condrictios, espectro trófico, nivel trófico

Massa \& Hozbor 2003). Además, como depredadores tope, cumplen un rol importante en el ecosistema y las modificaciones en su abundancia y distribución se reflejan de algún modo en la comunidad a la que pertenecen.

El género Schroederichthys pertenece a la familia Scyliorhinidae (Carcharhiniformes), una de las más numerosas entre los tiburones, que se distribuye en los océanos Atlántico, Pacífico e Índico y agrupa a unas cien 
especies (Cervigón \& Cousseau 1971, Compagno 1984, Menni et al. 1984, Lloris \& Rucabado 1991). En América del Sur esta familia incluye cuatro especies: S. tenuis Springer, 1966 (norte de Brasil), S. chilensis Guichenot, 1848 (sur de Chile y Perú), S. saurisqualus Soto, 2001 (sur de Brasil) y S. bivius Müller \& Henle, 1838 (sur de Brasil, Argentina y Chile) (Soto 2001). Esta última especie, que había sido citada como Halaelurus bivius por Menni et al. (1979), se distribuye específicamente en el Pacífico y en el Atlántico sudoccidental entre los $36^{\circ}$ y $54^{\circ} \mathrm{S}$, hasta profundidades de 350 m (Goztonyi \& Menni 1978, Menni \& Gosztonyi 1982, Van Der Molen et al. 1998), en el Golfo San Jorge (Sánchez \& Prenski 1996), dentro del Canal de Beagle (López et al. 1996) y alrededor de las Islas Malvinas (Laptikhovsky et al. 2001, Marí \& Sánchez 2002).

Antecedentes previos sobre la alimentación de la especie han sido presentados por Menni et al. (1979) para la región de la plataforma continental argentina, por Matallanas et al. (1993) para el canal de Beagle y por Laptikhovsky et al. (2001) para la región de Islas Malvinas. Todos estos autores le asignan un hábito trófico oportunista, con consumo de invertebrados (crustáceos y moluscos) y peces óseos de tamaño moderado, situación también mencionada para el género, en la recopilación realizada por Ebert et al. (1996).

En el Mar Argentino no existe pesca dirigida a pintarroja y como otros condrictios, es capturada en pesquerías pelágicas, demersales de altura y tangoneras, como fauna acompañante de la merluza común (Merluccius hubbsi Marini, 1933), la merluza de cola (Macruronus magellanicus Lönnberg, 1907), la polaca (Micromesistius australis Norman, 1937), el calamar (Illex argentinus Castellanos, 1960) y el langostino (Pleoticus muelleri Bate, 1888), tanto en buques comerciales, como en los buques de investigación pesquera (Angelescu \& Prenski 1987, Sánchez \& Prenski 1996, Wöhler et al. 1999, Gallardo 2006).

El objetivo de este trabajo fue analizar algunos aspectos biológico-pesqueros de la pintarroja, como la distribución geográfica, variaciones de su abundancia y alimentación, a partir de datos de campañas de investigación realizadas durante el período 1996-2006.

\section{Material y métodos}

La información utilizada en este estudio provino de cruceros de investigación realizados con los BIPs Cap. Oca Balda y Dr. Eduardo L. Holmberg, en el período comprendido entre 1996 y 2006. Se analizó información de 1924 lances de pesca, provenientes de dos áreas. Entre agosto y octubre (invierno-primavera) el área considerada entre $34^{\circ}$ y $48^{\circ} \mathrm{S}$, fue la de Plataforma Bonaerense y Norpatagónica, en adelante denominada PBN, con un rango de profundidades entre 49 y 346 m. Durante la época estival, entre febrero y marzo, se contó con información de la Plataforma Patagónica Austral entre $45^{\circ}$ y $54^{\circ} \mathrm{S}$, en adelante PPA, a profundidades entre los 45 y $650 \mathrm{~m}$ (Tabla 1). Cabe señalar que el área comprendida entre $45^{\circ}$ y $48^{\circ} \mathrm{S}$ fue visitada en ambas épocas. Para el análisis trófico se

\section{Tabla 1}

Valores anuales de abundancia relativa y biomasa de $S$. bivius estimados en el área de Plataforma Bonaerense y Norpatagónica (PBN), para el período 1996-2006. IC = Intervalos de confianza, 2002 = sin datos

Annual values of relative abundance and biomass of S. bivius estimated in Bonaerense and Northern Patagonian Shelf area $(\mathrm{PBN})$ among 1996-2006 period. IC = confidence intervals, 2002 = no data

\begin{tabular}{|c|c|c|c|c|}
\hline Año & $\begin{array}{l}\text { Abundancia } \\
\text { relativa media } \\
\quad\left(\mathrm{t} \mathrm{mn}^{-2}\right)\end{array}$ & IC $80 \%(+/-)$ & Biomasa (t) & IC $80 \%(+/-)$ \\
\hline 1996 & 0,13 & 0,02 & $11.968,71$ & $4.758,38$ \\
\hline 1997 & 0,10 & 0,02 & $8.391,26$ & $1.986,84$ \\
\hline 1998 & 0,08 & 0,02 & $7.074,25$ & $1.584,32$ \\
\hline 1999 & 0,14 & 0,03 & $15.644,59$ & $2.951,22$ \\
\hline 2000 & 0,17 & 0,04 & $19.997,31$ & $4.648,20$ \\
\hline 2001 & 0,12 & 0,03 & $14.679,72$ & $3.168,21$ \\
\hline 2003 & 0,10 & 0,02 & $11.520,82$ & $1.967,24$ \\
\hline 2004 & 0,12 & 0,03 & $9.401,76$ & $2.568,80$ \\
\hline 2005 & 0,24 & 0,03 & $26.239,05$ & $2.888,28$ \\
\hline 2006 & 0,19 & 0,07 & $12.970,11$ & $4.732,94$ \\
\hline
\end{tabular}


utilizó además información de campañas realizadas en 1993 y 1994. Asimismo, para comparar la distribución de la especie con años anteriores, se utilizaron datos propios obtenidos durante las campañas de prospección de los BIPs Walter Herwig y Shinkai Maru, llevadas a cabo en 1978 y 1979, en toda la plataforma.

Se estimaron los índices de abundancia total en toneladas (t) y abundancia relativa en toneladas por milla náutica cuadrada $\left(\mathrm{t} \mathrm{mn}^{-2}\right)$ por lance, para cada área y año del período analizado, utilizando para su cálculo el método de área barrida (Alverson \& Pereyra 1969), con intervalos de confianza del $80 \%$. Con tal fin se consideraron las capturas de la especie, el área barrida durante cada arrastre y las eficiencias relativas equivalentes entre los buques de características técnicas similares (Giussi et al. 2002).

De cada individuo muestreado se tomaron datos de longitud, peso, sexo y se revisó el contenido estomacal (a bordo y en tierra). Se analizaron y compararon las distribuciones de frecuencias de longitudes, las relaciones longitud-peso, la proporción de sexos y la alimentación en las regiones consideradas. Para describir y comparar la alimentación de la pintarroja en el Golfo San Jorge (GSJ), área costera entre $45^{\circ}-48^{\circ} \mathrm{S}$, de la cual no se obtuvo información trófica durante el presente trabajo, se reanalizaron datos utilizados por Sánchez \& Prenski (1996).

Se identificó cada ítem presa hasta la menor categoría taxonómica posible y se incluyó su nombre común siguiendo a Cousseau (1995), consignando su peso y longitud, cuando fue posible para determinar el espectro trófico específico. Para reconstruir el peso original de la presa en el momento de la ingestión, se utilizaron regresiones longitud total (LT) vs. peso total (P) existentes o comparación con los individuos 'no digeridos' de la misma especie presa. En el caso de presas muy digeridas se utilizó la regresión entre fragmentos encontrados en el estómago (telson, otolitos, etc.) y el peso total.

Se construyó el espectro trófico específico para cada zona geográfica utilizando el porcentaje en peso (\%P) de cada categoría taxonómica considerada. Para la comparación entre zonas se utilizó el índice alimentario (IA) de Lauzanne (1975, en Rosecchi \& Nouaze, 1987) donde: $\mathrm{IA}=(\% \mathrm{~F} x \mathrm{\% P}) / 100$, siendo $(\% \mathrm{~F})$ la frecuencia de ocurrencia y (\%P) el porcentaje en peso. Los valores de IA se categorizaron en función de su importancia relativa como: 1) alimento preferencial o dominante (AI > 50), 2) secundario $(25<$ IA $>50)$, frecuente $(10<$ IA $>25)$ y 4$)$ accidental u ocasional (IA < 10). Se construyó el espectro trófico específico para cada zona geográfica utilizando el porcentaje en peso $(\% \mathrm{P})$ de cada categoría taxonómica considerada.
Se calculó el nivel trófico (NT) según Cortés (1999) para cada una de las áreas.

$$
N T=1+\left(\sum_{j=1}^{n} P_{j} \times N T_{j}\right)
$$

donde: P peso y NT nivel trófico de la presa $j$

La amplitud de nicho trófico para cada área investigada fue calculada a partir del índice de diversidad de Shannon (Krebs 1989):

$$
H^{\prime}=\left(-\sum_{j=1}^{n} P_{j} * L n P_{j}\right)
$$

donde: $\mathrm{P} j$ es el peso del $j$-ésimo componente de la dieta por el total de pesos de la muestra.

Para determinar las variaciones del espectro trófico durante la ontogenia, se utilizó el análisis de correlación canónica (ACC) a partir de una matriz de porcentajes de frecuencia de ocurrencia de las presas $(\% \mathrm{~F})$ por clase de longitud total. Se calculó la variable canónica N1 como:

$$
\mathrm{N}_{1}=\sum \mathrm{C}_{\mathrm{i}} * 0 \% \mathrm{~F}_{\mathrm{i}}
$$

donde: $\mathrm{N}_{1}$ es la combinación lineal del $\% \mathrm{~F}_{\mathrm{i}}$ de cada presa (i) en el contenido estomacal, $\mathrm{C}_{i}$ es un coeficiente canónico, calculado de forma tal que $\mathrm{N}_{1}$ tenga máxima correlación con la longitud total. El ACC se realizó considerando dos grupos, uno constituido por una sola variable la longitud total del depredador, y otro donde las variables fueron los \%F de cada presa, siendo los resultados de este análisis interpretados como los de una regresión múltiple. Se graficó el valor de la variable canónica $\left(\mathrm{N}_{1}\right)$ vs. la longitud total (LT), y el punto de inflexión en esa recta indica un cambio en la dieta.

También se empleó el índice de solapamiento de Schöener (1974) para la comparación entre sexos y zonas:

$$
\mathrm{S}_{\mathrm{xy}}=1-0,5 \sum \mathrm{P}_{\mathrm{xi}}-\mathrm{P}_{\mathrm{yi}}
$$

donde: $\mathrm{P}_{\mathrm{xi}}$ : proporción de la presa $i$ en la dieta de los ejemplares $x$ a comparar, $\mathrm{P}_{\mathrm{yi}}$ : proporción de la presa $i$ en la dieta de los ejemplares $y$ a comparar. Valores superiores a 0,6 indican un alto solapamiento en la dieta. 


\section{Resultados}

\section{Distribución y abundancia}

En la Plataforma Bonaerense y Norpatagónica (PBN) se analizaron las capturas correspondientes a 928 lances positivos que representaron el 43,7\% del total de lances realizados durante el período invierno-primavera. La presencia de pintarroja se detectó entre 49 y 150 m, sin embargo, las mayores concentraciones se registraron de 90 a $110 \mathrm{~m}$, entre las latitudes $45^{\circ}$ y $47^{\circ} \mathrm{S}$ y a temperaturas de 6,3 a $7,8^{\circ} \mathrm{C}$.

La abundancia relativa por lance varió entre 0,01 y 2,86 t mn ${ }^{-2}$, aumentando progresivamente hacia el sur (Fig. 1a y b). En PBN, en 1998, se observó el valor mínimo de abundancia relativa media anual $\left(0,08 \mathrm{t} \mathrm{mn}^{-2}\right)$, los valores máximos $\left(0,24 t \mathrm{mn}^{-2}\right.$ y $\left.0,19 \mathrm{t} \mathrm{m}^{-2}\right)$ ocurrieron en los dos últimos años analizados (2005-2006). La biomasa total anual osciló entre 7.000 t en 1998 y 26.000 t en 2005 (Tabla 1).
En la Plataforma Patagónica Austral (PPA) se registraron 733 lances positivos, que representaron el 56\% del total realizado en verano. En esta región, la pintarroja se capturó entre los 45 y 199 m de profundidad y excepcionalmente apareció en un lance a $346 \mathrm{~m}$. Las abundancias relativas por lance oscilaron entre 0,02 y $9,11 \mathrm{t} \mathrm{mn}^{-2}$. Las abundancias relativas mayores a $4 \mathrm{t} \mathrm{mn}^{-2}$ se registraron en dos subáreas: la primera entre los $47^{\circ} \mathrm{y}$ $48^{\circ} \mathrm{S}$, a profundidades cercanas a $100 \mathrm{~m}$, con temperaturas de fondo entre 6,7 y $7,6^{\circ} \mathrm{C}$; la segunda se ubicó entre los $51^{\circ}$ y $53^{\circ} \mathrm{S}$, en una zona cercana a la costa, entre los 50-80 $\mathrm{m}$, con temperaturas de hasta $10,7^{\circ} \mathrm{C}$ (Fig. 2a y b).

En PPA, la abundancia relativa media anual evidenció una tendencia descendente entre $1997\left(0,68 \mathrm{t} \mathrm{mn}^{-2}\right)$ y 2003 $\left(0,19 \mathrm{t} \mathrm{mn}^{-2}\right)$. El máximo valor estimado de biomasa fue de 67.256 t en 1997, mientras que el mínimo fue de 18.636 t en 2003. Un notorio descenso se observó en la biomasa, a partir de 1999, y posteriormente una leve estabilización con oscilaciones anuales (Tabla 2).
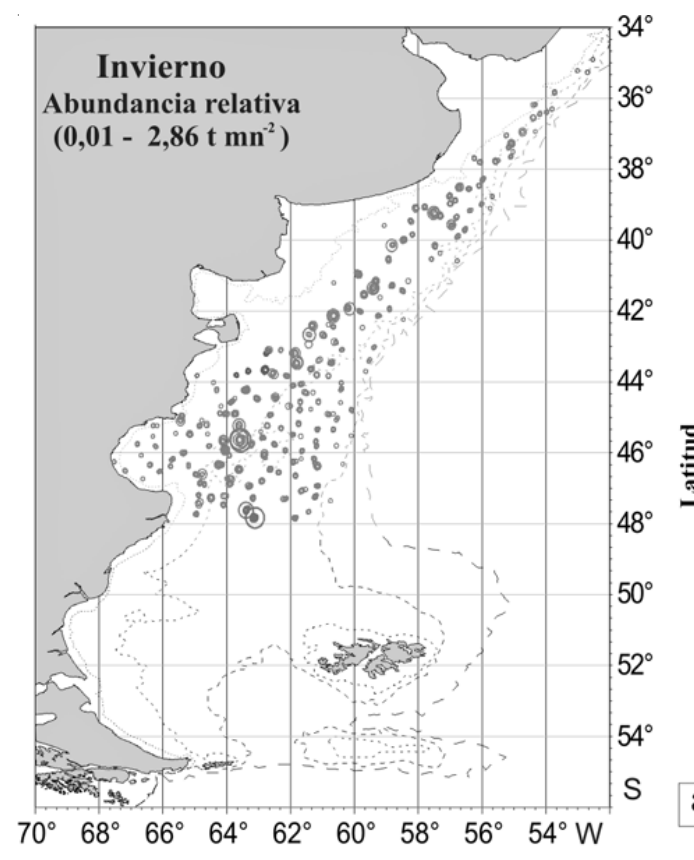

\section{Figura 1}

a) Ubicación geográfica y abundancia relativa $\left(\mathrm{t} \mathrm{mn}^{-2}\right)$ en cada uno de los lances de pesca con presencia de $S$. bivius, en PBN. b) Valores de abundancia relativa $\left(\mathrm{t} \mathrm{mn}^{-2}\right)$ en relación a latitud y profundidad para el período 1996-2006

a) Geographical allocation and relative abundance values $\left(\mathrm{t} \mathrm{nm}^{-2}\right)$ in each fishing trawl with S. bivius presence in PBN. b) Relative abundance values $\left(\mathrm{t} \mathrm{nm}^{-2}\right)$ in relation to latitude and depth, for the period 1996-2006 


\section{Tabla 2}

Valores anuales de abundancia relativa y biomasa de $S$. bivius estimados en el área de Plataforma Patagónica Austral (PPA), para el período 1997-2006. IC = Intervalos de confianza, $2002=\sin$ datos

Annual values of relative abundance and biomass of $S$. bivius estimated in Southern Patagonian Shelf area (PPA) for the period 1997-2006. IC = confidence intervals, $2002=$ no data

\begin{tabular}{ccccc}
\hline Año & $\begin{array}{c}\text { Abundancia } \\
\text { relativa media } \\
\left(\mathrm{t} \mathrm{mn}^{-2}\right)\end{array}$ & IC 80\%(+/-) & Biomasa (t) & IC 80\%(+/-) \\
\hline 1997 & 0,68 & 0,12 & $67.256,07$ & $12.429,76$ \\
1998 & 0,61 & 0,10 & $60.888,11$ & $10.431,45$ \\
1999 & 0,20 & 0,04 & $20.118,32$ & $3.612,39$ \\
2000 & 0,30 & 0,05 & $29.430,85$ & $4.970,22$ \\
2001 & 0,20 & 0,03 & $20.311,45$ & $2.750,53$ \\
2003 & 0,19 & 0,03 & $18.635,63$ & $3.308,44$ \\
2004 & 0,26 & 0,06 & $26.189,33$ & $5.529,01$ \\
2005 & 0,38 & 0,07 & $37.914,87$ & $7.313,65$ \\
2006 & 0,28 & 0,05 & $28.155,72$ & $5.204,67$ \\
\hline
\end{tabular}

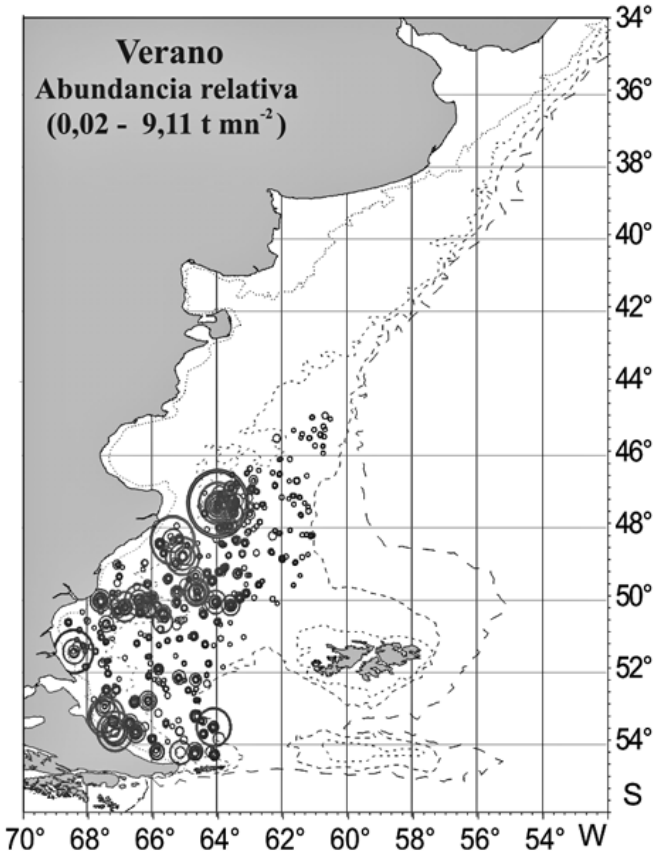

\section{Distribución de frecuencias de longitudes}

En PBN, el rango de longitudes observado de pintarroja fue de 32 a 78 cm LT; el tamaño máximo de las hembras fue de $67 \mathrm{~cm}$ LT y de los machos fue de $78 \mathrm{~cm}$ LT; además el 90\% de ellos fueron adultos, es decir mayores de $48 \mathrm{~cm}$ LT (Fig. 3a). En la PPA, el rango fue más amplio, la distribución de longitudes de los machos osciló entre 33$82 \mathrm{~cm}$ LT, mientras que las hembras presentaron un rango entre 35 y $78 \mathrm{~cm}$ LT. En ambas regiones se observaron modas similares para cada sexo: alrededor de $56 \mathrm{~cm} \mathrm{LT}$ para las hembras y entre 72 y $75 \mathrm{~cm}$ LT para los machos (Fig. 3a y b).

La proporción macho/hembra fue de 2,2/1 en PBN y de 1,5/1 en PPA, mientras que en el Golfo San Jorge, el $97 \%$ de los individuos fueron machos. Las estimaciones para la relación longitud (LT) y peso (P) realizadas en cada área y para el total de los individuos, resultaron ser similares, no detectándose diferencias significativas (Tabla 3).
Plataforma Patagónica Austral (PPA)

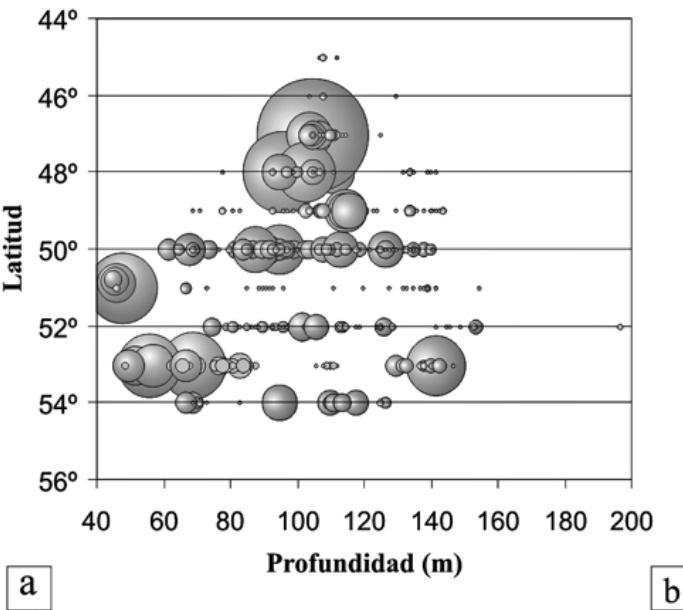

Figura 2

a) Ubicación geográfica y abundancia relativa $\left(\mathrm{t} \mathrm{mn}^{-2}\right)$ en cada uno de los lances de pesca con presencia de $S$. bivius, en PPA. b) Valores de abundancia relativa $\left(\mathrm{t} \mathrm{mn}^{-2}\right)$ en relación a latitud y profundidad para el período 1997-2006

a) Geographical allocation and relative abundance values $\left(\mathrm{t} \mathrm{nm}^{-2}\right)$ in each fishing trawl with S. bivius presence in PPA. b) Relative abundance values $\left(\mathrm{t} \mathrm{nm}^{-2}\right)$ in relation to latitude and depth, for the period 1997-2006 
PBN - invierno

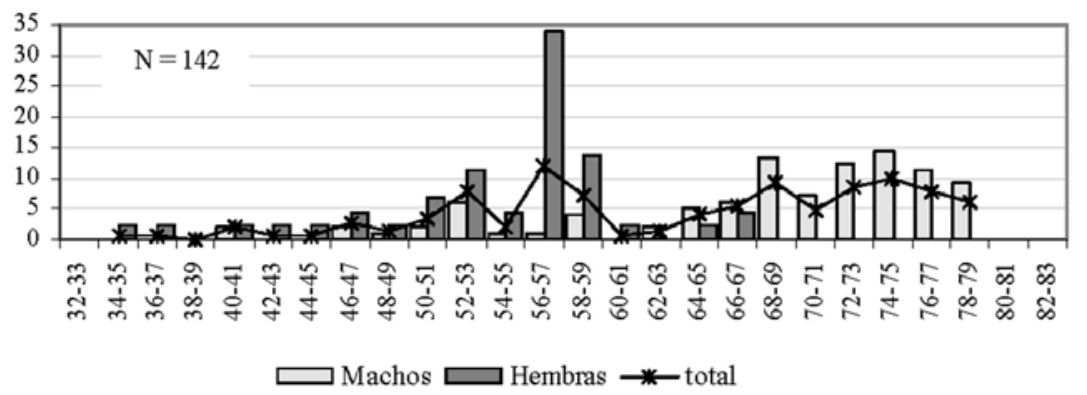

a)

PPA - verano

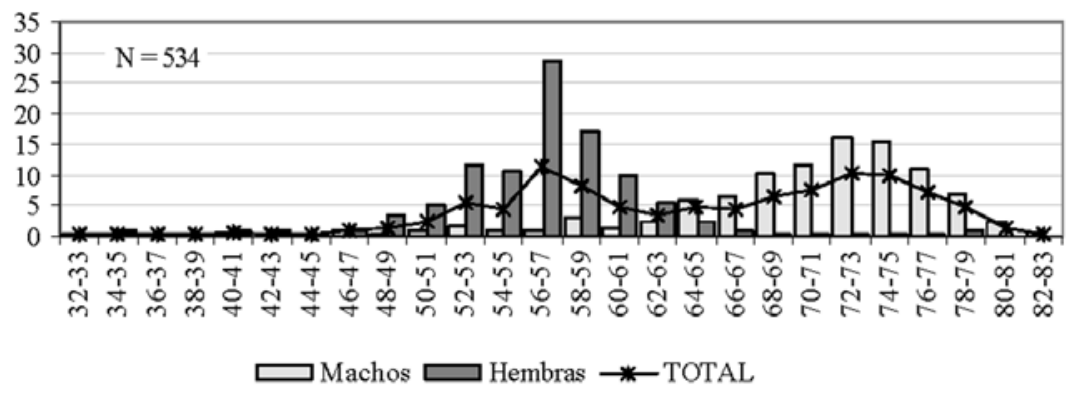

b)

Figura 3

Distribución de frecuencia de longitud de $S$. bivius en intervalos de $2 \mathrm{~cm}$ de LT en a) PBN y b) PPA

Length frequency distribution of $S$. bivius in $2 \mathrm{~cm}$ LT intervals in a) PBN and b) PPA

Tabla 3

Parámetros de la regresión largo-peso de S. bivius, para cada área considerada y para el total

Length-weight relationship parameters of S. bivius, estimated by each area and total

\begin{tabular}{ccccc}
\hline Área & $\mathrm{N}$ & $\mathrm{a}$ & $\mathrm{b}$ & $\mathrm{R}^{2}$ \\
\hline PBN & 142 & 0,007 & 2,774 & 0,908 \\
PPA & 469 & 0,008 & 2,736 & 0,906 \\
Total & 611 & 0,007 & 2,753 & 0,909 \\
\hline
\end{tabular}

El peso máximo observado de pintarroja en ambas zonas, correspondió a machos de $79 \mathrm{~cm} \mathrm{LT}$, que en PBN fue de $1.600 \mathrm{~g}$ y en PPA fue de $1.850 \mathrm{~g}$.

\section{Alimentación general}

Del total de 736 individuos analizados, el 88\% presentó alimento en el estómago. Al realizar la comparación entre sexos, el valor del índice de Schöener fue de 0,97, lo que indicaría un solapamiento total entre machos y hembras, por lo que la alimentación de la pintarroja fue analizada en conjunto.

A partir del análisis de correlación canónica se determinó un cambio en la alimentación alrededor de los 64 cm LT, evidenciado en el punto de inflexión de la recta (Fig. 4). El coeficiente de correlación canónica fue de 0,92, indicando un buen ajuste de los datos. Considerando este resultado, se analizó la dieta entre dos grupos de longitudes: entre 32-63 cm LT y entre 64-82 cm LT (Fig. 5). 
N1

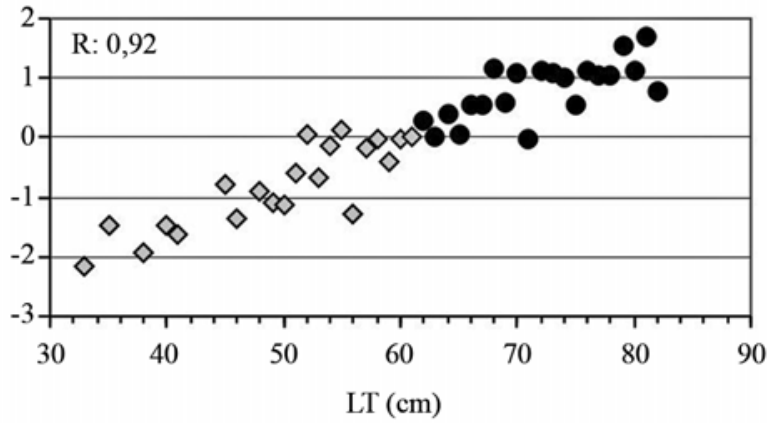

Figura 4

Relación entre largo total (LT cm) y la variable canónica (N1) para el total de los individuos de $S$. bivius analizados

Total length (LT cm) and canonical variable (N1) relationship on total $S$. bivius analyzed

Los crustáceos bentónicos estuvieron presentes en ambos grupos de talla de pintarroja, con poca variación en su frecuencia $(54 \% \mathrm{~F}$ y $52 \% \mathrm{~F}$ ) (Fig. 5), aunque con distintas especies y tamaños de la presa. Los individuos de pintarroja menores de $64 \mathrm{~cm}$ LT consumieron preferentemente decápodos peneidos (camarones) e isópodos de pequeño tamaño, mientras que los mayores, prefirieron decápodos Brachyura, como cangrejos Majidae y Peltarion spinosulum.

Entre las pintarrojas menores de $64 \mathrm{~cm}$ LT, fueron importantes en la dieta los invertebrados bentónicos, con 39 \%F. Entre ellos consumieron preferentemente poliquetos, holoturoideos, sipuncúlidos y actinias en orden de importancia (Fig. 5). Mientras que en los individuos mayores de $64 \mathrm{~cm}$ LT, se registró una marcada disminución de estos invertebrados (24\%F), representados casi exclusivamente por poliquetos principalmente de la familia Eunicidae.

Entre los moluscos, la presa dominante en los contenidos estomacales de ambos grupos de talla de pintarroja fueron los cefalópodos, representando un $30,2 \% \mathrm{~F}$ en la dieta de los individuos mayores de $64 \mathrm{~cm} \mathrm{LT}$, y un $14,8 \% F$ para los individuos de menores tallas (Fig. 5). Este grupo trófico estuvo integrado principalmente por el calamar (Illex argentinus), y por los pulpos (Octopus tehuelchus y Eledone spp.). Como presa ocasional y con similares valores de frecuencia para ambos grupos de longitudes, se encontró el calamarete (Loligo gahi).

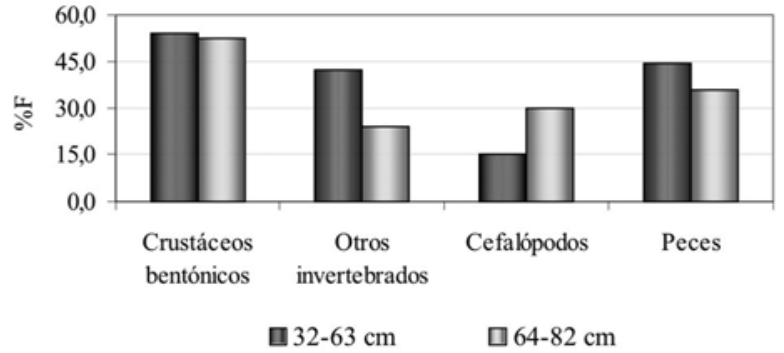

Figura 5

Frecuencia de ocurrencia $(\% \mathrm{~F})$ de los principales grupos de presas, para cada rango de longitud de $S$. bivius

Frequency of occurrence $(\% \mathrm{~F})$ of main prey groups in each length range of $S$. bivius

Los peces fueron una presa ligeramente más importante para los individuos de tallas menores de 64 cm LT $(44,5 \% \mathrm{~F})$, siendo dominantes los de pequeño tamaño, como anchoíta (Engraulis anchoita), mictófidos y Agonopsis. (Fig. 5). Las pintarrojas mayores de 64 cm LT consumieron las mismas especies de peces, aunque en menor proporción $(35,6 \% \mathrm{~F})$ y agregaron a su dieta, juveniles de peces de mayor tamaño como merluza (Merluccius hubbsi) y polaca (Micromesistius australis). La presencia de juveniles y adultos de nototenia (Patagonothotem ramsayi) fue similar en ambos grupos de tallas.

\section{Alimentación por regiones}

En la Plataforma Bonaerense y Norpatagónica (PBN), el 99\% de los 29 estómagos examinados de pintarroja presentó contenido estomacal, con 29 ítems presa; el índice de diversidad calculado fue 1,6 y el nivel trófico fue 3,06. Se identificaron dos grupos de ítems presa como 'frecuente': los peces, con 15,8 IA y los crustáceos bentónicos, con 12,6 IA(Tabla 4).

Los peces, con el 46,9\%P y el 33,7\%F, estuvieron representados principalmente por juveniles y adultos de merluza $(17,8 \% \mathrm{P})$ y de nototenia $(2,7 \% \mathrm{P})$. También se identificaron peces de pequeño tamaño como anchoíta (17,9\%P) y Raneya brasiliensis (1,3\%P) (Tabla 5).

Los crustáceos bentónicos representaron el 25,1\%P y 50\%F (Tabla 4). Entre los más abundantes se encontraron decápodos Brachyura como Libidoclaea granaria $14,8 \% \mathrm{P}$ y el cangrejo $P$. spinosulum $(8,3 \% \mathrm{P})$. 
Tabla 4

Principales grupos de presa (\%) en la dieta de $S$. bivius en las áreas consideradas (PBN, PPA y GSJ). $\mathrm{P}=$ peso, $\mathrm{F}=$ frecuencia, $\mathrm{IA}=$ índice alimentario. $\mathrm{N}=$ número de estómagos observados

Main prey groups (\%) in S. bivius diet in considered areas (PBN, PPA and GSJ). $\mathrm{P}=$ weight, $\mathrm{F}=$ frequency, $\mathrm{IA}=$ alimentary index. $\mathrm{N}=$ number of stomach observed

\begin{tabular}{lrrrrrrrrrr}
\hline \multirow{1}{*}{ Grupos presa } & \multicolumn{3}{c}{ PPA (N=607) } & \multicolumn{3}{c}{ PBN (N=129) } & \multicolumn{3}{c}{ GSJ (N=204) } \\
& \multicolumn{1}{c}{$\mathrm{P}$} & $\mathrm{F}$ & $\mathrm{IA}$ & $\mathrm{P}$ & $\mathrm{F}$ & IA & P & F & IA \\
\hline \multirow{2}{*}{ Crustáceos bentónicos } & 5,4 & 25,8 & 1,4 & 25,1 & 50,0 & 12,6 & 42,8 & 55,7 & 23,8 \\
Otros invertebrados bentónicos & 4,0 & 20,8 & 0,8 & 1,0 & 5,9 & 0,1 & 8,4 & 21,7 & 1,8 \\
Moluscos cefalópodos & 79,1 & 24,6 & 19,5 & 26,9 & 9,9 & 2,7 & 11,5 & 7,1 & 0,8 \\
Peces & 12,0 & 26,0 & 3,1 & 46,9 & 33,7 & 15,8 & 37,2 & 15,6 & 5,8 \\
Zooplancton gelatinoso & 0,0 & 1,0 & $<0,1$ & - & - & - & - & - & - \\
Macroalgas & 0,1 & 1,0 & $<0,2$ & - & - & - & - & - & - \\
\hline
\end{tabular}

Otros grupos de crustáceos encontrados, aunque escasamente representados $(<1 \% \mathrm{P})$, fueron los decápodos Anomura, bogavantes, camarones, isópodos y anfípodos gamáridos, entre otros (Tabla 5).

Los moluscos cefalópodos fueron considerados alimento 'ocasional' con un 2,7 IA, y representaron el 26,9\% y 9,9\%F. La presa más importante dentro de este grupo fue el calamar Illex argentinus (26,1\%), mientras que el calamarete y los pulpitos fueron casuales (Tablas 4 y 5).

Los 'otros invertebrados bentónicos' fueron, considerados un alimento 'ocasional' con valores de 0,1 IA $(1,0 \% \mathrm{P}$ y $5,9 \% \mathrm{~F})$, destacándose entre ellos los poliquetos y los equinodermos (erizos, ofiuros y estrellas de mar) (Tablas 4 y 5).

El 83\% de los 607 estómagos de pintarroja examinados en la Plataforma Patagónica Austral (PPA) presentó contenido estomacal, con 51 ítems presa. En individuos de esta área se obtuvo el menor valor del índice de diversidad (1,319), mientras que el nivel trófico de 3,3 fue el mayor calculado.

Si bien los porcentajes de frecuencia fueron similares para los primeros cuatro grupos de presas, los cefalópodos se destacaron con elevados valores (79,1\%P y $24,6 \% \mathrm{~F})$, por lo tanto fueron considerados presa 'frecuente' con un 19,5 IA (Tabla 4). Este grupo estuvo integrado principalmente por el calamar (I. argentinus) (69,7\%P). El resto de los cefalópodos estuvo escasamente representado e incluyó al calamarete (L. gahi), los pulpitos (Eledone sp. y O. tehuelchus) y el calamarcito Semirossia tenera (Tabla 5).
Los crustáceos bentónicos fueron 'ocasionales' con un 1,4 IA, representando el 25,8\%F y el 5,4\%P (Tabla 4). Dentro de este grupo se destacan los cangrejos Majidae (3\%) entre los que se identificó principalmente Libidoclaea granaria. Con porcentajes en peso inferiores a $1 \% \mathrm{P}$ se encontraron Munida spp., P. spinosulum y algunos isópodos de los géneros Cirolana, Serolis, Idotea y de la familia Anthuridae. Los decápodos Natantia identificados en esta región fueron Pleoticus muelleri, Austropandalus grayi, Campylonotus vagans y Callianassa brachyophtalma (Tabla 5).

El total de los peces consumidos representó el 12\%P y el $26 \% \mathrm{~F}$, con un 3,1 IA, siendo entonces este grupo considerado alimento 'ocasional' (Tabla 4). Entre ellos se identificaron las nototenias ( $P$. ramsayi) con $7,3 \% \mathrm{P}$ y Myxine sp. con 1,1\%P. La pintarroja también consumió ocasionalmente especies de gadiformes $(<1 \% \mathrm{P})$ como juveniles de merluza, polaca, viuda, bacalao austral y merluza de cola (Tabla 5). Además se encontraron restos de peces que por su grado de digestión no pudieron ser identificados.

El grupo de otros invertebrados bentónicos fue 'ocasional' según el valor de 0,8 IA, con el 4\%P y el 20,8\%F (Tabla 4). Los poliquetos (2\%P) fueron los mas abundantes. Se identificaron Aphrodita longicornis y ejemplares de las familias Eunicidae, Onuphidae y Terebellidae. Cabe destacar que el peso de estos individuos fue posiblemente subestimado dada su labilidad. El resto de las presas incluidas en este grupo presentaron $<1 \% \mathrm{P}$, en orden de importancia fueron los sipunculidos, priapulidos, echiuridos, equinodermos y celenterados bentónicos (Tabla 5). 


\section{Tabla 5}

Espectro trófico cualitativo y cuantitativo de S. bivius para las áreas PBN, PPA y GSJ, expresado en porcentaje en peso

S. bivius qualitative and quantitative trophic spectrum in PBN, PPA and GSJ areas, expressed as percentage of weight

\begin{tabular}{|c|c|c|c|c|}
\hline \multirow[b]{2}{*}{ Nombre científico } & \multirow[b]{2}{*}{ Nombre común } & \multicolumn{3}{|c|}{ Área } \\
\hline & & 总 & 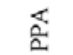 & $\overline{0}$ \\
\hline \multicolumn{5}{|l|}{ Talofitas } \\
\hline Macrocystis sp. & algas & & 0,01 & \\
\hline \multicolumn{5}{|l|}{ Phylum Cnidaria } \\
\hline Celenterados & coloniales & & 0,03 & \\
\hline Actinaria & anémona & & 0,03 & \\
\hline \multicolumn{5}{|l|}{ Phylum Chaetognata } \\
\hline Sagitta sp. & gelatinoso & & 0,01 & \\
\hline \multicolumn{5}{|l|}{ Phylum Annelida } \\
\hline Aphrodita longicornis & ratón de mar & & 0,03 & \\
\hline Nereis & poliqueto & & & 0,12 \\
\hline $\begin{array}{l}\text { Polychaeta (Eunicidae, } \\
\text { Onuphidae, Terebellidae) }\end{array}$ & poliqueto & 0,45 & 1,96 & 6,50 \\
\hline \multicolumn{5}{|l|}{ Phylum Mollusca } \\
\hline Nuculana sulculata & bivalvo & & & 0,07 \\
\hline Marginella warrenii & pie de caracol & & 0,07 & \\
\hline Illex argentimus & calamar & 26,10 & 69,70 & 5,70 \\
\hline Loligo gahi & calamarete & 0,35 & 2,10 & \\
\hline Octopus tehuelchus & pulpo & & 1,84 & 4,86 \\
\hline Eledone spp. & pulpito & & 0,64 & \\
\hline Semirossia tenera & calamarcito & 0,05 & 1,90 & \\
\hline \multicolumn{5}{|l|}{ Superclase Crustacea } \\
\hline Amphipoda Gammariidea & anfipodo gamárido & $<0,01$ & 0,03 & \\
\hline Themisto gaudichaudii & anfipodo hipérido & $<0,01$ & $<0,01$ & \\
\hline Euphausia spp. & eufáusido & & & $<0,01$ \\
\hline Decapoda & spp. NI & & 0,01 & \\
\hline Pleoticus muelleri & langostino & 0,14 & 0,17 & 10,61 \\
\hline Peisos petrunkevitchi & camaroncito & 0,02 & & \\
\hline Caridea & camaroncito NI & & & 0,10 \\
\hline Campylonotus spp. & camarón & & $<0,01$ & \\
\hline Austropandalus grayi & camarón & 0,10 & 0,10 & \\
\hline $\begin{array}{l}\text { Callianassa } \\
\text { brachyophtalma }\end{array}$ & camarón & & 0,03 & \\
\hline Brachyura & cangrejos NI & 0,20 & 2,10 & 2,20 \\
\hline Peltarion spinosulum & cangrejo & 8,30 & 0,90 & 5,40 \\
\hline Majidae & cangrejo araña & 14,80 & 2,90 & 10,50 \\
\hline Libidoclaea granaria & cangrejo araña & 0,16 & & 1,30 \\
\hline Paralomis spp. & centollón & 0,20 & & \\
\hline Pagurus spp. & cangrejo ermitaño & 0,16 & 0,03 & \\
\hline Eurypodius latreillei & cangrejo araña & & 0,01 & \\
\hline
\end{tabular}

\begin{tabular}{|c|c|c|c|c|}
\hline \multirow[b]{2}{*}{ Nombre cientifico } & \multirow[b]{2}{*}{ Nombre común } & \multicolumn{3}{|c|}{ Área } \\
\hline & & 恕 & $\overleftarrow{a}$ & $\vec{S}$ \\
\hline Eurypodius latreillei & cangrejo araña & & $<0,01$ & \\
\hline Munida spp. & bogavante & 0,60 & 0,30 & 1,80 \\
\hline Pterygosquilla armata a. & estomatópodo & & 0,40 & 9,30 \\
\hline Cirolana sp. & isópodo & $<0,01$ & 0,05 & \\
\hline Idotea $\mathrm{sp}$. & isópodo & & 0,06 & \\
\hline Serolis sp. & isópodo & 0,01 & 0,06 & \\
\hline Anthuridae & isópodo & & 0,08 & \\
\hline
\end{tabular}

Phylum Echinodermata

erizo $\quad 0,40$

Ophiuroidea

ofiuro

0,02

Asteroidea

estrella de mar

0,10

Phylum Echiura

equiuridos

Phylum Priapula

priapulidos

0,90

Phylum Sipuncula

sipunculidos

1,01

Phylum Urochordata

Salpa sp.

gelatinoso

0,25

Phylum Chordata

Ascidiacea

ascidia

$0,01<0,01$

Clase Cyclostomi

Myxine sp.

(A) y huevos

0,37

$1,45 \quad 11,10$

Clase Chondrichthyes

Schroederichthys bivius pintarroja (J)

0,36

Rajidae raya NI $(J)$

Clase Osteichthyes

restos NI

$6,40 \quad 3,40 \quad 3,90$

Engraulis anchoita

anchoita

17,90

Gymnoscopelus nicholsi

mictófido

$0,24 \quad 0,03$

Macruromus

merluza de cola

(J)

$<0,01$

Merluccius hubbsi

merluza común

17,90

0,08

Salilota australis

bacalao austral (J)

0,10

Raneya brasiliensis

1,32

Illuocoetes fimbriatus

viuda

0,10

Cottoperca gobio

cotoperca $(\mathrm{J})$

0,04

Patagonothoten ramsayi nototenia

$2,70 \quad 7,30$

0,30

Sebastes occulatus cabrilla (J)

0,01

Agonopsis chiloensis

$0,12 \quad 0,32$

Symphurus spp.

lengüita

0,50

Xistreuris rasile lenguado $(\mathrm{J})$

0,10

Micromesistiusaustralis polaca 
Esporádicamente también se encontró zooplancton gelatinoso (salpas y quetognatos) escasamente representado $(0,02 \%$ y y $1,0 \%$ F) (Tabla 4$)$.

En esta región y como alimento raro se encontraron restos de macroalgas (Macrocystis sp.) en los contenidos estomacales (0,11\%P y 1\%F) (Tablas 4 y 5$)$.

En el Golfo San Jorge (GSJ), el 90\% de los 204 estómagos de pintarroja analizados presentó contenido estomacal (Sánchez \& Prenski 1996). Se identificaron 22 ítems presa y el valor del índice de diversidad fue 2,56 siendo el valor más alto de las tres regiones. El nivel trófico para esta región fue 3,06 similar al encontrado en PBN.

El grupo de presas mas importante fue el de los crustáceos bentónicos con un 23,8 IA (42,8\% y y 55,7\%F) considerado alimento 'frecuente' (Tabla 4). En este grupo predominaron los estomatópodos (Pterigosquilla armata armata) con $9,4 \% \mathrm{P}$ y los decápodos (33\%P). Entre estos últimos se destacan especies de la familia Majidae, el langostino ( $P$. muelleri), el cangrejo $P$. spinosulum y Munida gregaria (Tabla 5).

Los peces representaron el 5,8 IA (37,2\%P y 15,6\%F), destacando la merluza (21\%P), Myxine (11\%P) y $R$. brasiliensis (5\%P); el resto presentó valores inferiores al $1 \% \mathrm{P}$ (Tablas 4 y 5 ).

Los moluscos encontrados que representaron en total $11,5 \% \mathrm{P}$ y $7,1 \% \mathrm{~F}$ (0,8 IA), fueron los cefalópodos $I$. argentinus y $O$. tehuelchus y como alimento raro se encontraron ejemplares del bivalvo Nuculana sulculata (Tablas 4 y 5 ).

En pintarrojas del GSJ se observó la mayor proporción de 'otros invertebrados bentónicos', con 8,4\%P y 21,7\%F (1,8 IA). En este grupo se destacaron los poliquetos Nereidae y Eunicidae, los equiuridos y las ascidias (Tablas 4 y 5).

El nivel trófico medio de la pintarroja para las tres regiones fue de 3,25. Al comparar los índices alimentarios (IA) de los grandes grupos tróficos (Tabla 4) entre las áreas, los valores obtenidos del índice de Schöener fueron: $\mathrm{S}_{\text {PPA-PBN }}=0,793, \mathrm{~S}_{\text {PPA-GSJ }}=0,776 \mathrm{y} \mathrm{S}_{\text {PBN-GSJ }}=0,875$. Este resultado indica que la dieta de la pintarroja presenta solapamiento entre las tres áreas consideradas.

\section{Discusión}

\section{Distribución y abundancia}

La pintarroja presentó una amplia área de distribución, ya que fue capturada en aguas de plataforma desde $34^{\circ}$ hasta $54^{\circ} \mathrm{S}$, a profundidades entre 45 y $199 \mathrm{~m}$. Coincidiendo con nuestros resultados, en la campaña de prospección pesquera realizada por BIP Shinkai Maru en 1978, se capturó esta especie, mencionada como Halaelurus vibius, en toda la plataforma entre los 28 y 179 m de profundidad y hasta los 55ㅇ (Menni et al. 1979, Gosztonyi 1979, Gosztonyi 1981). También Otero et al. (1982) mencionan que esta especie habita el sector de aguas profundas al norte del paralelo de $48^{\circ} \mathrm{S}$, durante el verano y el invierno, a profundidades entre 50 y $200 \mathrm{~m}$. Estudios mas recientes (Van der Molen et al. 1998) indican la presencia de la especie como captura incidental o 'by-catch' de las pesquerías costeras patagónicas, entre $41^{\circ}-51^{\circ} \mathrm{S}$, durante todo el año.

La abundancia relativa máxima de pintarroja por lance de pesca fue casi tres veces mayor en la Plataforma Patagónica Austral $\left(9,1 \mathrm{t} \mathrm{mn^{-2 } )}\right.$ que el máximo registrado en la Plataforma Bonaerense y Norpatagónica $\left(2,86\right.$ t mn $\left.{ }^{-2}\right)$ en todo el período analizado. Entre las latitudes $45^{\circ}-48^{\circ} \mathrm{S}$ se observó la presencia de la pintarroja en las dos épocas consideradas (febrero-marzo y agosto-octubre) con elevadas abundancias relativas (Figs. 1 y 2). Esto concuerda con resultados obtenidos en el presente trabajo, con datos de los cruceros de investigación realizados por el BIP Walther Herwig en 1978, cuando los mayores rendimientos se obtuvieron entre los $44^{\circ}$ y $53^{\circ} \mathrm{S}$, en los meses de invierno. Así mismo, Otero et al. (1982), para una región comprendida entre los $39^{\circ} \mathrm{S}$ y $48^{\circ} \mathrm{S}$, mencionan abundancias relativas para invierno de $0,42 \mathrm{t}$ $\mathrm{mn}^{-2}$ y para el verano de $0,36 \mathrm{t} \mathrm{mn}^{-2}$ y una biomasa total de 22.000 t. Como se observa, los valores de abundancia en esa época eran significativamente mayores que los que hemos observado en años posteriores en PBN.

En el presente trabajo, las máximas abundancias relativas de pintarroja se detectaron a temperaturas entre $6^{\circ}$ y $8^{\circ} \mathrm{C}$, quizás debido a la preferencia de la especie por aguas mas frías como mencionaran también Gosztonyi (1973) y Bellisio et al. (1979). Este último autor la define como una especie característica de la corriente de Malvinas, que transporta aguas subantárticas con valores de temperatura entre $4^{\circ}$ y $6^{\circ} \mathrm{C}$. Esta preferencia también explicaría su presencia alrededor de las Islas Malvinas (Laptikhovsky et al. 2001, Marí \& Sánchez 2002), en el Canal de Beagle (López et al. 1996) y en la denominada Provincia Magallánica (Menni et al. 1979, Menni 1983) con temperaturas entre 4 y $14^{\circ} \mathrm{C}$.

Al analizar los valores anuales de biomasa y abundancia relativa, se observó a partir del año 1999, una variación con tendencia opuesta para cada área, es decir un aumento de la biomasa en PBN y una disminución en PPA (Tablas 1 y 2). Esto podría estar relacionado con una disminución del esfuerzo pesquero en PBN a partir de 1999, cuando la flota comercial arrastrera dirigida a 
Merluccius hubbsi, que captura pintarroja como fauna acompañante, trasladó su operatividad y esfuerzo al sur de los $48^{\circ} \mathrm{S}$ (Resolución SAGPyA No 24/99). Esta variación de las biomasas se evidenció en otras especies de condrictios que también integran las capturas comerciales como fauna acompañante (Marí 2005).

\section{Distribución de frecuencias de longitudes}

En ambas regiones se observaron modas similares en la distribución de frecuencias de longitudes para cada sexo. Las hembras, presentaron un rango de LT más acotado con una moda alrededor de los $56 \mathrm{~cm} \mathrm{LT}$, en tanto que para los machos el rango fue mas amplio y la moda se presentó a mayores longitudes, entre 72 y $75 \mathrm{~cm} \mathrm{LT} \mathrm{(Fig.}$ 3a y b). Los machos fueron de mayor tamaño que las hembras, aunque a igual longitud, las hembras tuvieron mayor peso. Estas diferencias coinciden con lo manifestado por Menni et al. (1979). Entre los Scyliorhinidae, los machos pueden alcanzar un tamaño igual o mayor que las hembras de la misma especie, mientras que en el resto de los tiburones son las hembras las que a menudo alcanzan mayores longitudes que los machos (Flamang et al. 2008).

La relación macho/hembra fue 2,2/1 en PBN y 1,5/1 en PPA, corroborando estos resultados la segregación por sexos que presenta esta especie en plataforma, tal como fue mencionado por Menni et al. (1979) y Compagno (1984). También se pudo confirmar esta segregación en el Golfo San Jorge, a partir de nuestra posterior revisión de la base de datos de Sánchez \& Prenski (1996), donde se pudo consignar que el $97 \%$ de los individuos presentes eran machos adultos (55-79 cm de LT).

Las estimaciones para la relación longitud (LT) vs. peso (P) realizadas por sexos y para el total de los individuos en cada área, resultaron ser similares, no detectándose diferencias significativas.

\section{Alimentación general}

A partir de los resultados obtenidos en el presente trabajo, la pintarroja puede ser definida como un depredador demersal-bentónico, de hábitos preferentemente carnívoros y macrófagos, con un amplio espectro trófico y un comportamiento de tipo oportunista. Dichos resultados fueron obtenidos también por Menni et al. (1979), Matallanas et al. (1993), Ebert et al. (1996) y Laptikhovski et al. (2001).

No encontramos diferencias significativas entre la alimentación de machos y hembras, aunque la pintarroja presenta un marcado dimorfismo sexual tanto en la forma de la boca como en la dentición (Gosztonyi 1973). También
Matallanas et al. (1993) mencionan que no hay correlación entre la morfología de la boca y los dientes, con la dieta de machos y hembras. Esto es común entre otros elasmobranquios y dentro de la familia Scyliorhinidae se ha observado que el tipo de dentición de los machos está relacionada al comportamiento sexual, ya que durante la pre-cópula retienen a la hembra mordiéndola, para facilitar la inserción del clasper y mantenerla en la postura adecuada que asegure la entrada de esperma (Pratt \& Carrier 2005).

En este estudio también se encontraron variaciones en la dieta en relación al desarrollo ontogénico, estableciéndose un cambio en la misma a los $64 \mathrm{~cm}$ LT. Aunque los cambios ontogenéticos en la dieta ocurren en casi todos los peces, generalmente en tamaños próximos a la primera madurez, esto no fue observado para la pintarroja, dado que la madurez sexual de las hembras ocurre alrededor de los $40 \mathrm{~cm}$ LT, y en los machos alrededor de los 53 cm LT (Gosztonyi 1973).

Los individuos menores y mayores de $64 \mathrm{~cm} \mathrm{LT}$, consumieron los mismos grandes grupos tróficos aunque con diferente frecuencia de aparición. Asímismo, se observó una relación directa entre el tamaño del depredador y el de las presas consumidas, donde los depredadores de mayor tamaño prefirieron presas mayores, especialmente del grupo de los crustáceos bentónicos. Resultados similares fueron obtenidos por Laptikhovsky et al. (2001) en la región de las Islas Malvinas, mencionando un cambio en la dieta, desde pequeños crustáceos planctónicos a grandes crustáceos bentónicos en los individuos de mayor tamaño. Sin embargo, diferimos con estos autores, tanto en la talla donde éstos identifican la inflexión en la dieta (40 cm LT), como en la importancia que le asignan a los crustáceos planctónicos. En nuestro estudio, la presencia de estos crustáceos (eufáusidos y anfípodos hipéridos), fue rara $\mathrm{y}$ en valores inferiores al 0,001\%P. Asimismo, no hay mención de la presencia de crustáceos planctónicos en la dieta de la pintarroja, en otros trabajos de investigación.

\section{Alimentación por regiones}

En las regiones analizadas, la pintarroja mostró una marcada tendencia por los hábitos demersal-bentónicos, pero dado su carácter de especie oportunista presentó un amplio espectro trófico, consumiendo en cada región aquellos grupos de presas más abundantes (Tabla 4). Se comprobó que la dieta de la pintarroja presenta solapamiento entre las tres áreas consideradas, principalmente debido a la presencia de crustáceos bentónicos en diferentes proporciones. Cabe señalar que en PPA, fue conspicua la presencia de cefalópodos Illex 
argentinus en su dieta (Sánchez \& Marí 2005). El calamar habita mas frecuentemente entre los $35^{\circ} \mathrm{S}$ y $52^{\circ} \mathrm{S}$ en influencia de aguas templado frías de origen subantártico, con un área de alta concentración durante el verano entre los $43^{\circ} \mathrm{S}-48^{\circ} \mathrm{S}$ (Brunetti et al. 1998) coincidente con PPA.

En PBN, en los contenidos estomacales de la pintarroja aparecieron principalmente juveniles de merluza y peces de pequeño tamaño como anchoita y nototenia, presentes durante todo el año en el área. Aquí se registró un caso excepcional de canibalismo de un juvenil, no mencionado por otros autores para esta especie y poco habitual para los tiburones de pequeño tamaño. La mayoría de los casos de canibalismo en condrictios se han reportado para grandes tiburones (Heithaus 2004).

Finalmente, en el Golfo San Jorge la pintarroja consumió mayormente macrocrustáceos bentónicos, muy abundantes y con alta diversidad en dicha área (Sánchez \& Prenski 1996). También en la región del Canal Beagle, Matallanas et al. (1993) mencionan que el régimen alimentario de la pintarroja fue principalmente carcinófago. Esto también se observó para $S$. chilensis y $S$. bivius en las costas chilenas, donde ambas especies superponen su distribución (Fariña et al. 1993).

La presencia de restos de macroalgas en los contenidos de las hembras puede considerarse como accidental, dado que se trata de una especie de hábitos carnívoros. Sin embargo, como las hembras de pintarroja depositan sus huevos en las frondas de estas algas pardas, como está documentado para otros Scyliorhinidae (Compagno 1984, Fariña \& Ojeda 1993, Ellis \& Shackley 1997), quizás accidentalmente las ingieran al alimentarse de otras presas que utilizan estas algas de refugio.

El nivel trófico de $S$. bivius para el espectro trófico general fue de 3,25, valor ligeramente inferior al mencionado por Cortés (1999) de 3,8. El valor obtenido por este autor parece muy alto para la especie, quizás por haber sido calculado en base a grandes grupos tróficos y con un número de individuos reducido, mientras que en nuestro trabajo se utilizó el nivel trófico aportado por cada item presa y para un elevado número de ejemplares muestreados.

Podemos concluir que la pintarroja presentó una amplia dispersión geográfica e integra la fauna acompañante de la mayoría de las pesquerías de arrastre de la plataforma continental argentina. Residente en el área de estudio durante todo el año, su amplio espectro trófico y su característica de oportunista, le permite utilizar los recursos disponibles en un variado rango de profundidades. Dada la escasa información actualizada sobre esta especie, nuestro trabajo representa un importante aporte al conocimiento de la misma.

\section{Agradecimientos}

Agradecemos a los evaluadores anónimos de este manuscrito, los valiosos y constructivos comentarios sobre nuestro trabajo, que permitieron enriquecerlo y mejorarlo.

\section{Literatura citada}

Alverson D \& W Pereyra. 1969. Demersal fishery explorations in the Northeastern Pacific Ocean. An evaluation of exploratory fishing methods and analytical approaches to stock size and yield forecasts. Journal of the Fisheries Research Board of Canada 26(8): 1985-2001.

Angelescu V \& LB Prenski. 1987. Ecología trófica de la merluza común (Merluccius hubbsi) del Mar Argentino. Parte 2. Dinámica de la alimentación analizada sobre la base de las condiciones ambientales, la estructura y las evaluaciones de los efectivos en su área de distribución. Contribución INIDEP 561: 1-205.

Bellisio NB, RB Lopez \& A Torno. 1979. Peces marinos patagónicos, 279 pp. Publicación Subsecretaría de Pesca, Buenos Aires.

Brunetti NE, ML Ivanovic \& B Elena. 1998. Calamares omastréfidos (Cephalopoda, Ommastrephidae). En: Boschi E (ed). El mar Argentino y sus recursos pesqueros. 2. Los moluscos de interés pesquero. Cultivos y estrategias reproductivas de bivalvos y equinoideos, pp. 37-68. Instituto Nacional de Investigación y Desarrollo Pesquero, Mar del Plata.

Cervigón F \& MB Cousseau. 1971. Catálogo sistemático de la colección ictiológica del Instituto de Biología Marina. Instituto de Biología Marina, Contribución 169: 1-123.

Compagno LJV. 1984. FAO Species catalogue. 4. Sharks of the World. An annotated and illustrated catalogue of shark species known to date. 1 . Hexanchiformes to Lamniformes. FAO Fisheries Synopsis 125 4(1): 1-249.

Cortés E. 1999. Standardized diet compositions and trophic levels of sharks. ICES Journal of Marine Science 56: 707717.

Cousseau MB. 1995. Peces, crustáceos y moluscos registrados en el sector del Atlántico Sudoccidental comprendido entre $34^{\circ}$ y $55^{\circ} \mathrm{S}$, con indicación de las especies de interés pesquero. Informe Técnico, INIDEP 5: 1-96.

Ebert DA, PD Cowley \& LJV Compagno. 1996. A preliminary investigation of the feeding ecology of catsharks (Scyliorhinidae) off the west coast of southern Africa. South African Journal of Marine Science 17: 233-240. 
Fariña JM \& PD Ojeda. 1993. Abundance, activity, and trophic patterns of the redspotted catshark, Schroederichthys chiliensis, on the Pacific temperate coast of Chile. Copeia 1993: 545-549.

Flammang BE, DA Ebert \& GM Cailliet. 2008. Reproductive biology of deep-sea catsharks (Chondrichthyes: Scyliorhinidae) in the eastern North Pacific. Environmental Biology of Fish 81: 35-49.

Gallardo MA. 2006. Pesca incidental de tiburones en la pesquería de altura del langostino patagónico (Pleoticus muelleri). Tesis de grado, Facultad de Ciencias Naturales, Universidad Nacional de la Patagonia San Juan Bosco, Trelew, 45 pp.

Giussi AR, NR Marí \& F Sánchez. 2002. Estimación de la biomasa y composición de longitudes de la merluza de cola a partir de campañas estivales Período 1992 a 2002. Informe Técnico Interno, INIDEP 18: 1-12.

Gosztonyi A. 1973. Sobre el dimorfismo sexual secundario en Halaelurus bivius (Müller y Henle, 1841) Garman, 1913 (Elasmobranchii, Scyliorhinidae) en aguas patagónicofueguinas. Physis 32(85): 317-323.

Gosztonyi AE. 1981. Resultados de las investigaciones ictiológicas de la campaña I del B/I ‘Shinkai Maru’ en el Mar Argentino. Campañas de investigación pesquera en el Mar Argentino por los B/I 'Shinkai Maru' y 'Walther Herwig’ y el B/I ‘Marburg’, Años 1978 y 1979. Resultados de la parte Argentina Secc. IV. Estudios especiales del ecosistema marino. Contribuciones Científicas, INIDEP 383: 254-266.

Heithaus MR. 2004. Predator-prey interactions. En: Carrier JC, JA Musick \& MR Heithaus (eds). Biology of sharks and their relatives 17: 487-521. CRC Press, Boca Raton.

Krebs CJ. 1989. Ecological methodology, 654 pp. Harper Collins Publishers, New York.

Laptikhovsky VV, AI Arkhipkin \& AC Henderson. 2001. Feeding habits and dietary overlap in spiny dogfish Squalus acanthias (Squalidae) and narrow-mouthed catshark Schroederichthys bivius (Scyliorhinidae). Journal of the Marine Biological Association of the United Kingdom 81(6): 1015-1018.

Lloris D \& J Rucabado. 1991. Ictiofauna del Canal de Beagle (Tierra del Fuego), aspectos ecológicos y análisis biogeográfico. Publicación Especial, Instituto Español de Oceanografía 8: 1-182.

López HL, ML García \& NA San Román. 1996. Lista comentada de la ictiofauna del Canal Beagle, Tierra del Fuego, Argentina. Publicación Especial, CADIC, Contribución Científica 23: 1-84.

Marí NR. 2005. Síntesis de la información derivada de las campañas de evaluación estival de especies demersales australes, desarrolladas en el Mar Argentino, entre los $45^{\circ}$ y $54^{\circ} \mathrm{S}$, por los buques del INIDEP, durante el período 1992 al 2001. Peces cartilaginosos. Informe Técnico Interno INIDEP 93: 1-30.

Marí NR \& F Sánchez. 2002. Espectros tróficos específicos de varias especies de peces demersales de la región austral y sus variaciones anuales entre 1994 y 2000. Informe Técnico Interno, INIDEP 88: 1-9.

Massa A \& N Hozbor. 2003. Peces cartilaginosos de la plataforma argentina, explotación, situación y necesidades para un manejo pesquero adecuado. Frente Marítimo 19: 199-206.

Massa AM, NR Marí, AR Giussi \& N Hozbor. 2007. Índices de abundancia de Squalus acanthias en la Plataforma Continental Argentina. Informe Técnico Interno, INIDEP 06/07: 1-18

Matallanas J, M Carrasson \& M Casadevall. 1993. Observations on the feeding habits of the narrow-mouthed catshark Schroederichthys bivius (Chondrichthyes, Scyliorhinidae) in the Beagle Channel. Cybium 17(1): 5561.

Menni RC \& AE Gosztonyi. 1982. Benthic and semidemersal fish association in the argentine sea. Studies on Neotropical Fauna and Environment 17: 1-29.

Menni RC, AE Gostonyi \& H Lopez. 1979. Sobre la ecología y biología de Halaelurus bivius (Chondrichthyes, Scyliorhinidae). Revista del Museo Argentino de Ciencias Naturales, Ecología 2(3): 71-88.

Menni RC, RA Ringuelet \& RHL Aramburu. 1984. Peces marinos de Argentina y Uruguay, 359 pp. Editorial Hemisferio Sur, Buenos Aires.

Otero HO, SI Bezzi, MA Renzi \& GA Verazay. 1982. Atlas de los recursos pesqueros demersales del Mar Argentino. Contribución INIDEP 423: 1-248.

Pratt HL Jr \& JC Carrier. 2005. Elasmobranch courtship and mating behavior. En: Hamlett WC (ed). Reproductive biology and phylogeny of Chondrichthyes, Sharks, Batoids and Chimaeras, pp. 129-164. Science Publishers, Enfield.

Pratt HL Jr \& JG Casey. 1990. Shark reproductive strategies as limiting factors in directed fisheries, with a review of Holden's method of estimating growth parameters. En: Pratt HL Jr, SH Gruber \& T Taniuchi (eds). Elasmobranchs as living resources: Advances in the biology, ecology, systematic, and status of the fisheries. NOAA Technical Report 90: 97-110.

Rosecchi E \& Y Nouaze. 1987. Comparaison de cinq indices alimentaires utilisés dans l'analyse des contenus stomacaux. Revue des Travaux de l' Institut des Pêches Maritimes 49(3/4): 111-123.

Sánchez F \& NR Marí. 2005. Interacciones tróficas entre especies de peces demersales en la región austral entre $45^{\circ} \mathrm{S}$ y 54오. Informe Técnico Interno, INIDEP 91: 1-9. 
Sánchez F \& LB Prenski. 1996. Relaciones tróficas multiespecíficas en la pesquería del Golfo San Jorge. Revista de Investigación y Desarrollo Pesquero 10: 57-71.

Schöener TW. 1974. Resource partitioning in ecological communities. Science 185: 27-39.

Soto JMR. 2001. Schroederichthys saurisqualus sp. nov., (Carchariniformes, Scyliorhinidae) a new species of catshark from southern Brazil, with further data on Schroederichthys species. Mare Magnum 1(1): 37-50.
Van der Molen S, G Caillet \& R González. 1998. By catch of sharks in Patagonian coastal trawl fisheries. Marine and Freshwater Research 49(7): 641-644.

Wöhler OC, AR Giussi, SB García de la Rosa, F Sánchez, JE Hansen, HD Cordo, GA Alvarez-Colombo, S Incorvaia, R Reta \& VE Abachian. 1999. Resultados de la campaña de evaluación de peces demersales australes efectuada en el verano de 1997. Informe Técnico, INIDEP 24: $1-60$.

Recibido el 31 de octubre de 2008 y aceptado el 29 de mayo de 2009 